\title{
O026: Countrywide prevalence study of healthcare-associated infections in brazilian hospitals: preliminary results
}

\author{
CMCB Fortaleza ${ }^{1 *}$, MC Padoveze ${ }^{2}$, C Kiffer $^{3}$, AL Barth ${ }^{4}$, ICRS Carneiro ${ }^{5}$, JLN Rodrigues ${ }^{6}$, L Santos Filho ${ }^{7}$, MJG Mello ${ }^{8}$, \\ MD Asensi ${ }^{9}$, PP Gontijo Filho ${ }^{10}$, MS Pereira ${ }^{11}$, M Rocha $^{9}$, RS Kuchenbecker $^{12}$, ES Medeiros ${ }^{13}$, ACC Pignatari ${ }^{13}$, \\ IRAS - BRASIL ${ }^{1}$
}

From 2nd International Conference on Prevention and Infection Control (ICPIC 2013)

Geneva, Switzerland. 25-28 June 2013

\section{Introduction}

The knowledge of burden of Healthcare-Associated Infections (HAI) in hospitals is essential to drive governmental strategies for its prevention and control.

\section{Objectives}

To identify the prevalence of HAI in a representative sample of Brazilian hospitals.

\section{Methods}

A team of trained nurses carried out a hospital-wide HAI point prevalence survey in 2012. A sample of hospitals from five Brazilian regions was evaluated $(n=91$; total of 8,853 beds).

\section{Results}

The overall infection rate was $11.1 \%$, varying from $2.5 \%$ (hospitals with $<50$ beds) to $18.3 \%$ (hospitals with $>200$ beds). Reference hospitals showed $11.2 \%$ of overall infection rate. The most prevalent infections were pneumonia (3.6\%), bloodstream infection (3.5\%), surgical site infection (1.4\%), urinary tract infection $(1.1 \%)$ and skin infection $(0.4 \%)$. Hospitals with $>200$ beds were likely to have higher HAI rates $(\mathrm{RR}=1.71 ; \mathrm{IC}=1.398-2.10$; $\mathrm{P}<0.001)$. The risk factors more frequently identified were: central venous catheter $(17.8 \%)$, surgery $(15.5 \%)$, urinary catheter $(14.0 \%)$, and mechanical ventilators (8.1\%). Etiologic agents were identified only in 9.1\% (43/ 473) of infections. Gram-negative organisms were more

${ }^{1}$ Tropical Diseases, Faculdade de Medicina de Botucatu - UNESP - Univ Estadual Paulista, Botucatu, Brazil

Full list of author information is available at the end of the article frequent (56.0\%), among them, Klebsiella spp (19.0\%) and Pseudomonas aeruginosa (16\%) and were predominant. Among Gram-positives (35.0\%), coagulase-negative Staphylococci were more prevalent (16\%) than Staphylococcus aureus $(9.0 \%)$ or Enteroccoccus spp (6\%). Yeasts were identified in $9.0 \%$ of HAI.

\section{Conclusion}

These preliminary results emphasize both the relevance and the heterogeneity of HAI in Brazilian hospitals.

\section{Disclosure of interest}

None declared.

\begin{abstract}
Author details
${ }^{1}$ Tropical Diseases, Faculdade de Medicina de Botucatu - UNESP - Univ Estadual Paulista, Botucatu, Brazil. ²Public Health, Escola de Enfermagem USP - Universidade de São Paulo, Brazil. ${ }^{3}$ Laboratório Especial de Microbiologia Clínca, Universidade Federal de São Paulo, São Paulo, Brazil. ${ }^{4}$ Universidade Federal do Rio Grande do Sul, Porto Alegre, Brazil. ${ }^{5}$ UFPA Universidade Federal do Pará, Belém, Brazil. ${ }^{6}$ UFC - Universidade Federal do Ceará, Fortaleza, Brazil. ${ }^{7}$ UFPB - Universidade Federal da Paraíba, João Pessoa, Brazil. ${ }^{8}$ IMIP - Pernambuco, Recife, Brazil. ${ }^{9} \mathrm{FIOCRUZ} \mathrm{-} \mathrm{Rio} \mathrm{de} \mathrm{Janeiro,} \mathrm{Rio} \mathrm{de}$ Janeiro, Brazil. ${ }^{10}$ Microbiology, UFU - Universidade Federal de Uberlandia, Uberlandia, Brazil. ${ }^{11}$ UFGO - Universidade Federal de Goiás, Goiânia, Brazil. ${ }^{12}$ UFRGS - Universidade Federal do Rio Grande do Sul, Porto Alegre, Brazil.

${ }^{13}$ UNIFESP - Escola Paulista de Medicina, São Paulo, Brazil.
\end{abstract}

Published: 20 June 2013

doi:10.1186/2047-2994-2-S1-O26

Cite this article as: Fortaleza et al:: 0026: Countrywide prevalence study of healthcare-associated infections in brazilian hospitals: preliminary results. Antimicrobial Resistance and Infection Control 2013 2(Suppl 1):O26. 